\title{
Preference index-based allocation of optimized cropping area at the Mirpurkhas subdivision: Jamrao irrigation scheme in Sindh, Pakistan
}

\begin{abstract}
Optimal utilization of limited water resources is important due to the continuously increasing demand for it. This study was aimed at devising a model and a preference index for allocating limited land and water resources at the Mirpurkhas subdivision of the Jamrao irrigation scheme in Sindh, Pakistan. The deterministic linear programming (DLP) model formulated was set to the minimum and maximum area constraints and applied for different scenarios (at levels of $100,70,60$, and $50 \%$ of existing water supply), in an effort to find the optimum conditions of land area allocation. Emphasis was given to the cultivation of vegetable, banana, and oilseed crops during times of shortages of water. Upon optimization with the DLP model, the proposed preference index was found to be an effective guide in equitable distribution among the competing command areas through the resulting competitive and conflict-free allocating of the resources. The model cum index that was developed is proposed to be adopted while formulating the policy regarding land and water resources allocation during reduced canal flows at the Mirpurkhas subdivision of the Jamrao irrigation scheme at Sindh, Pakistan.
\end{abstract}

